Crop Breeding and Applied Biotechnology 15: 162-168, 2015

Brazilian Society of Plant Breeding. Printed in Brazil

\title{
ARTICLE
}

http://dx.doi.org/10.1590/1984-70332015v15n3a28

\section{Differential transformation efficiency of Japonica rice varieties developed in northern China}

\author{
Dan $\mathrm{Li}^{1}$, Hai Xu${ }^{1}$, Xiaoxue Sun ${ }^{1}$, Zhibo Cui ${ }^{1}$, Yuan Zhang ${ }^{1}$, Yuguan $\mathrm{Bai}^{2}$, Xiaoxue Wang ${ }^{1 *}$ and Wenfu Chen ${ }^{1}$
}

Received 13 December 2014

Accepted 19 January 2015

\begin{abstract}
The production of japonica rice in northern China plays an important role in food security for the world. Agrobacteriummediated gene transfer is becoming a powerful approach to generate germplasm and develop varieties. However, the transgenic efficiency of the japonica rice varieties in northern China has been completely unknown, which obstructs the development of transgenic breeding and the exploration of gene functions. In this study, the transgenic efficiencies of six japonica rice varieties developed in northern China are evaluated. The rates of primary and secondary callus induction of the varieties are similar. However, transgenic efficiency and the regeneration ability of the varieties are greatly different. The results have established a platform for transformation of the rice varieties and proposed a suitable variety, SN9816, for gene transfer. SN9816 can be applied as an elite germplasm for transgenic breeding and basic research of molecular biology in northern China or an area in the same latitude.
\end{abstract}

Key words: Agrobacterium-mediated transformation, transformation efficiency, japonica rice in northern China, Shen Nong 9816.

\section{INTRODUCTION}

Rice (Oryza sativa L.), which is cultivated in more than 115 countries and feeds over one third of the population worldwide, is one of the world's most important crops (Khush 1997, Raimondi et al. 2014). More than $90 \%$ of the world's rice is grown and consumed in Asia, where 60\% of the earth's people live. Rice is also one of the major staple foods in China, a center of origin of Asian cultivated rice (Khush 1997, Londo et al. 2006). The production of japonica rice in northern China is famous for its higher yield and good quality and accounts for $50 \%$ of the total production. Enhancing the yield and improving the quality of rice through breeding are effective ways of responding to increasing food demands. Molecular breeding, including marker assistant selection (MAS), transgenic breeding, and genome-wide association study (GWAS) provides breeders with new challenges and opportunities to increase the efficiency of selection, shorten the breeding period, and generate new germplasm with improved traits, which are difficult to improve by conventional methods (Jung et al. 2008).

Improvement of rice varieties through transferring genes or cis-acting elements from rice, plants, or other organisms to rice to confer new agronomic traits in rice, termed transgenic breeding, is an effective way to create new germplasm and breed new varieties (McLaren 2005). Agrobacterium-mediated transformation is a general method for genetic modification in many plant species because it allows efficient insertion of stable, unrearranged, single-copy sequences into plant genomes (Nishimura et al. 2006). Efficient methods for Agrobacterium-mediated transformation of japonica rice have been developed (Hiei et al. 1994, Hiei et al. 1997, Hiei and Komari 2008). Two japonica varieties, Nipponbare and Kitaake (developed in Japan), are good genotypes for Agrobacterium-mediated transformation. Based on this method, Agrobacteriummediated transformation methods have been improved, with minor modification for many rice varieties, not only japonica but also indica and tropical japonica (Nishimura et al. 2006, Hiei and Komari 2008, Saika and Toki 2010).

It has been demonstrated that transformation efficiencies are genotype dependent (Hiei et al. 1997, Hiei and Komari 2008). Although efficient procedures for Agrobacteriummediated transformation of either japonica or indica rice have been developed, the transgenic efficiency of the rice varieties developed in northern China have been completely

\footnotetext{
${ }^{1}$ Rice Research Institute, Key Laboratory of Northeast Rice Biology and Breeding, Ministry of Agriculture; Key Laboratory of Northern Japonica Super Rice Breeding, Ministry of Education; Shenyang Agricultural University, Shenyang 110866, China. *E-mail: xiaoxuewang6@163.com

${ }^{2}$ Northeast Yucai School, Shenyang 110179, China
} 
unknown (Hiei and Komari 2008, Saika and Toki 2010). Additionally, because of the higher latitude in northeast China and the sensitivity of rice heading to day length, Nipponpare could not successfully develop seed in the field under normal conditions. Even though Kitaake could be used to replace Nipponbare in northern Asia, it was hard for breeders to use Kitaake as breeding germplasm because the plant size of Kitaake is smaller than desired and its agronomic traits are not as good as expected. These factors restrict the development of transgenic breeding and the performance of molecular biological study in northeast China. In this study, five varieties which are popularly cultivated in northern China are used as explants to compare their response to Argobacterium-mediated transformation by using Nipponbare as control. Our studies have established a platform for Agrobacterium-mediated transformation of the japonica rice varieties developed in northern China and proposed a suitable variety, Shen Nong 9816, for Agrobacterium-mediated gene transfer with a transgenic efficiency and regeneration rate similar to Nipponbare.

\section{MATERIAL AND METHODS}

\section{Plant materials}

Six varieties of japonica-type rice were used in the study, including Shen Nong 9816 (SN9816), Shen Nong 265 (SN265), M31, Liao Xing 1 (LX1), Toyonishiki (Toyo, from Japan), and Nipponbare (Nipp, from Japan, sequenced). Among them, SN9816, SN265, M31, LX1, and Toyonishiki are widely cultivated in northern China, which is the major region for japonica rice production in China. Nipponbare was used as control, the seeds of which were produced in the Experimental Station of the Chinese Academy of Agricultural Science, Beijing (lat 39 92' N, long 116 46' E). The mature seeds of the other five varieties used for callus induction were produced in the Experimental Station of the Rice Research Institute at Shenyang Agricultural University (lat $41^{\circ} 80^{\prime} \mathrm{N}$, long $123^{\circ} 38^{\prime} \mathrm{E}$ ) in northeast China.

\section{Binary vector and Agrobacterium-mediated transformation}

The pCambia 1301 binary vector was applied, harboring the expression cassettes of the 35S:intron-GUSA gene (tagged by hexaHis), the $35 \mathrm{~S}$ : aadA gene (conferring kanamycin resistant to bacterium), and the $35 \mathrm{~S}$ : hptII gene (conferring hygromycin resistance to callus or plantlets) (Figure 1). The binary vector was transformed into the Agrobacterium strain EHA 105 through the Freeze-Thaw method.

A procedural representation of Agrobacterium-mediated transformation using secondary callus is shown in Figure
2. Dehulled mature seeds, which were sterilized with $70 \%$ ethanol for 1 minute and then $2.5 \%$ sodium hypochlorite for 20 minutes (Figure 2A), were inoculated in callus induction N6D medium (Figure 2B), containing $3.99 \mathrm{~g} \mathrm{~L}^{-1}$ Chu N6 Basal Medium with Vitamins (Phyto Technology Laboratories, Product No. C167), $0.1 \mathrm{~g} \mathrm{~L}^{-1}$ myo-inositol, $0.3 \mathrm{~g} \mathrm{~L}^{-1}$ casamino acids, $2.878 \mathrm{~g} \mathrm{~L}^{-1}$ proline, $2 \mathrm{mg} \mathrm{L}^{-1} 2$, 4-dichlorophenoxyacetic acid (2, 4-D), $30 \mathrm{~g} \mathrm{~L}^{-1}$ sucrose, and $5 \mathrm{~g} \mathrm{~L}^{-1}$ agar, $\mathrm{pH}=5.8$. Three-week-old secondary calli (Figure 2D) derived from 7-day-old primary calli (Figure 2C) were subcultured in fresh N6D medium for 3 days before co-cultivation with Agrobacterium (Figure

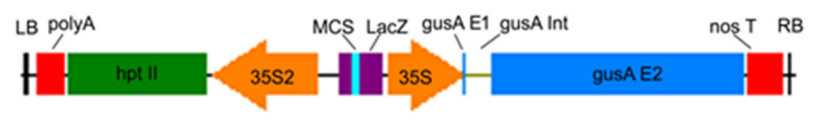

Figure 1. A map of pCambia 1301 binary vector between the left border and right border. LB: left border repeat; polyA: CaMV 3' UTR polyA signal; hpt II: hygromycin resistance gene; $35 \mathrm{~S} 2$ : CaMV $35 \mathrm{~S}$ promoter duplicated; MCS: multiple cloning site; Lac Z: Lac Z promoter and Lac Z alpha fragment; 35S: CaMV 35S promoter; gusA E1: gusA first exon; gusA Int: gusA intron; gusA E2: gusA second exon; nos T: nopaline synthase 3'UTR polyA signal; RB: right border repeat.
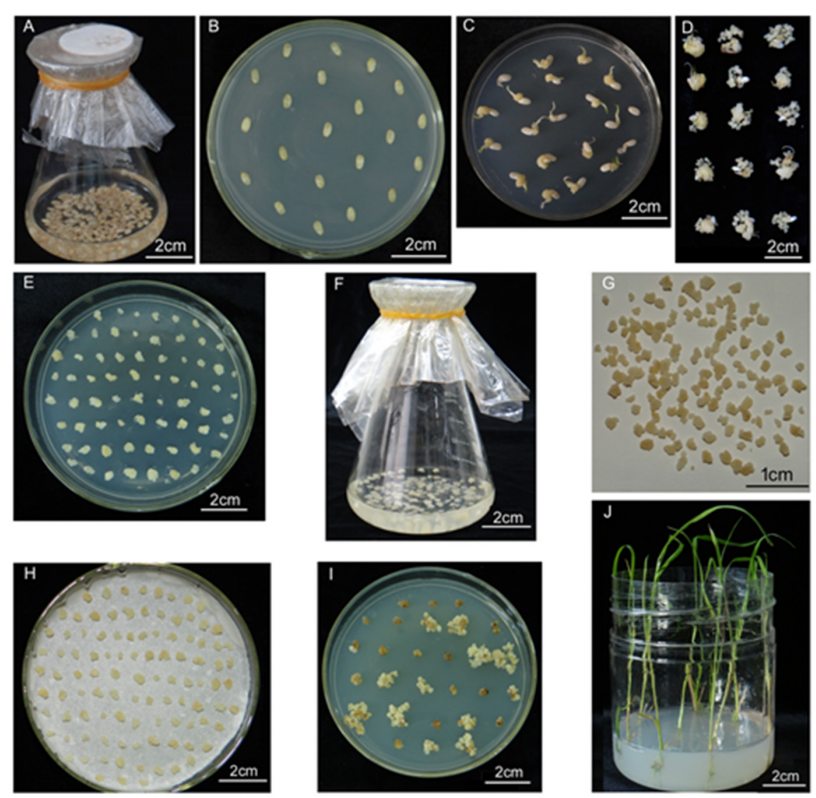

Figure 2. Procedures of Agrobacterium-mediated transformation for japonica rice varieties in northern China. (A) Sterilization of Seeds. (B) Inoculation of seeds in N6D medium. (C) Formation of primary calli in N6D medium. (D) Induction of secondary calli in N6D medium. (E) Preculture of the secondary calli in N6D medium three days before transformation. (F) Infection of the preculture secondary calli with the Agrobacterium harboring pCambia 1301 vector. (G) Drying of the infected calli before co-cultivation. (H) Co-cultivation with the Agrobacterium for three days in the dark. (I) Proliferation of Hyg-tolerant calli on the secondary selection medium, N6D-S medium. (J) Regeneration of transgenic shoot in root generation medium, MS-HF. The white or black line is the scale bar, representing $2 \mathrm{~cm}$ or $1 \mathrm{~cm}$ as indicated. 
2E). Agrobacterium co-cultivation followed the modified protocol described in (Toki et al. 2006). The infected and air-dried secondary calli (Figures $2 \mathrm{~F}, \mathrm{G}$ ) were co-cultivated with Agrobacterium for 3 days under constant darkness in 2N6-AS medium (Figure 2H), containing $3.99 \mathrm{~g} \mathrm{~L}^{-1} \mathrm{Chu} \mathrm{N} 6$ Basal Medium with Vitamins, $0.1 \mathrm{~g} \mathrm{~L}^{-1}$ Myo-inositol, 0.3 $\mathrm{g} \mathrm{L}^{-1}$ casamino acids, $2 \mathrm{mg} \mathrm{L}^{-1}$ 2, 4-dichlorophenoxyacetic acid (2, 4-D), $30 \mathrm{~g} \mathrm{~L}^{-1}$ sucrose, $10 \mathrm{~g} \mathrm{~L}^{-1}$ glucose, $5 \mathrm{~g} \mathrm{~L}^{-1}$ agar, and $1 \mathrm{ml}$ of $100 \mathrm{mMAS}, \mathrm{pH}=5.2$. The calli were then washed with water and cephalothin solution, and cultured in the N6D medium containing $50 \mathrm{mg} \mathrm{L}^{-1}$ hygromycin B (Hyg) (Roche Diagnostics, Basel, Switzerland, Product No. 10843555001) and $450 \mathrm{mg} \mathrm{L}^{-1}$ cephalothin for 10 days (N6D-S medium). After 10 days of selection, calli were transferred to fresh N6D-S medium for another 10 days (Figure 2I). For regeneration, calli growing vigorously on Hyg were transferred to MS-NK medium, containing 4.43 $\mathrm{g} \mathrm{L}^{-1}$ MS Basal Medium (Phyto Technology Laboratories, Product No. M519), $0.1 \mathrm{~g} \mathrm{~L}^{-1}$ Myo-inositol, $2 \mathrm{~g} \mathrm{~L}^{-1}$ casamino acids, $0.20 \mathrm{mg} \mathrm{L}^{-1}$ alpha-naphthalene acetic acid (NAA), 2 mg L-1 kinetin, $30 \mathrm{~g} \mathrm{~L}^{-1}$ sucrose, $30 \mathrm{~g} \mathrm{~L}^{-1}$ sorbitol, $30 \mathrm{mg} \mathrm{L}^{-1}$ Hyg, and $450 \mathrm{mg} \mathrm{L}^{-1}$ cephalothin, $\mathrm{pH}=5.8$, and cultured for more than 4 weeks. Shoots arising from callus in MS-NK medium were transferred to MS-HF medium, containing $4.43 \mathrm{~g} \mathrm{~L}^{-1} \mathrm{MS}$ Basal Medium, $0.1 \mathrm{~g} \mathrm{~L}^{-1}$ Myo-inositol, and $30 \mathrm{~g} \mathrm{~L}^{-1}$ sucrose, $\mathrm{pH}=5.8$ with $25 \mathrm{mg} \mathrm{L}^{-1} \mathrm{Hyg}$, and $450 \mathrm{mg}$ $\mathrm{L}^{-1}$ cephalothin to allow vigorous root growth (Figure $2 \mathrm{~J}$ ).

\section{GUS staining approach}

Transient expression of 35S:intron-GUS was assayed after 3 days of co-cultivation through detection of histochemical $\beta$-glucuronidase (GUS) reporter activity with 5-bromo-4-chloro-3-indolylglucuronide (X-Gluc, Thermol Scientific Cat\# R0851) as a substrate (Jefferson et al. 1987).

\section{Methods used in statistical analysis}

Three independent biological replicates were performed. The data were analyzed using the GraphPad Prism 5 software. The Tukey multiple comparison test of one-way analysis of variance (ANOVA) was used to detect the significance of differences.

\section{Genomic DNA extraction and PCR analysis}

For extraction of genomic DNA, rice seedlings were harvested, immediately frozen in liquid $\mathrm{N}_{2}$, and stored at $-80 \square$. Genomic DNA was extracted through the $2 \times$ CTAB method. PCR analysis to detect the hpt gene was performed by using rTaq DNA polymerase (Takara Biotechnology, Cat\# R500Z) with the primer pair of forward primer, HygF CTTCTGCGGGCGATTTGT, and reverse primer, HygR

\section{CAGCGTCTCCGACCTGAT.}

\section{RESULTS AND DISCUSSION}

\section{Callus formation abilities are similar in the varieties}

It is known that some rice varieties are tissue-culture sensitive, especially the indica rice varieties. To evaluate the ability for callus formation, sterilized mature seeds of the varieties were inoculated in N6D medium and grown for 7 days or 3 weeks. The status of primary callus growth, the rate of primary or secondary callus induction, and the rate of secondary callus growth were observed (Figure 3).

Yellow, compacted primary callus can be induced from all six varieties, but the emergence of primary calli from Liao Xing 1 (LX1), M31, and Shen Nong 265 (SN265) was later and slower than that of Nipponbare (Nipp), Shen Nong 9816 (SN9816), and Toyonishiki (Toyo) (Figure 3A). The rates of primary or secondary callus induction of the varieties were similar (Figures 3B, C). No significant difference in the rate of primary or secondary callus induction of the varieties was analyzed. However, a highly significant difference in the fresh weight of 100 callus cluster of the secondary calli (FWSC) after 15 days of subculture was observed among
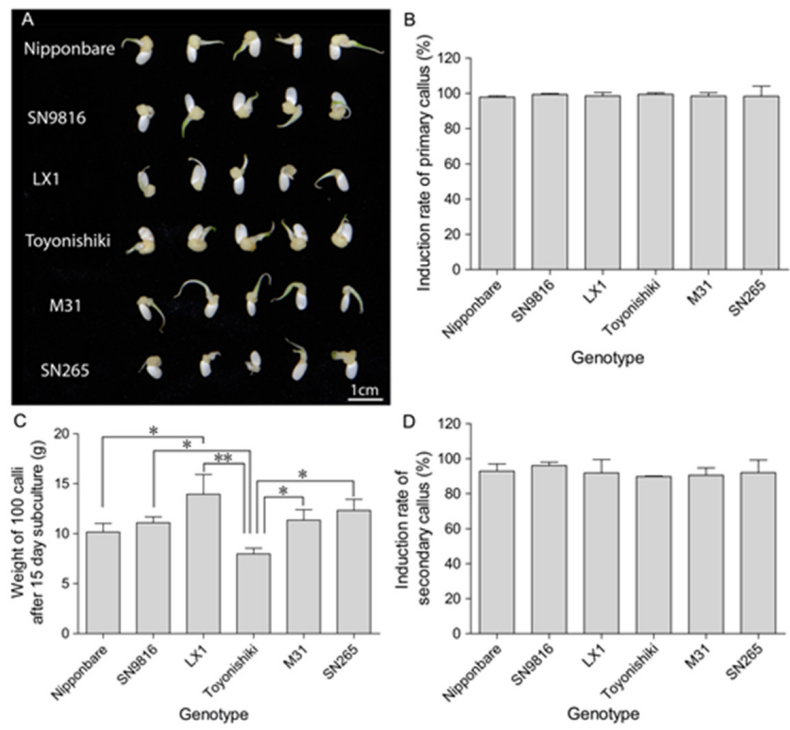

Figure 3. Callus formation of japonica rice varieties in northern China. (A) The 7-day-old primary calli of the varieties. (B) The rate of primary callus induction of the varieties. (C) The rate of secondary callus induction of the varieties. (D) The fresh weight of 100 callus cluster of secondary calli after 15 days of subculture. The white line is the scale bar, representing $1 \mathrm{~cm}$. The values are the mean and standard deviation (SD) from three independent biological experiments. SN9816: Shen Nong 9816; LX1: Liao Xing 1; SN265: Shen Nong 265. One asterisk $(*)$ represents significant difference $(P<0.05)$. Two asterisks $(* *)$ represent highly significant difference $(P<0.01)$. 
the varieties (Figure 3D). The FWSC of LX1 (13.94g) was the highest, and that of Toyo $(7.97 \mathrm{~g})$ was the lowest. The FWSC of LX1 was significantly higher than that of Nipp (10.16g). The FWSC of LX1 (13.94g), SN265 (12.33g), M31 (11.36g), or SN9816 (11.09g) was significantly higher than that of Toyo. However, no significant difference of the FWSC was detected between Nipp and SN9816, between Nipp and Toyo, and among SN9816, LX1, M31, and SN265. These results indicated that the secondary calli induced from the five varieties developed in northern China are of good status for Agrobacterium-mediated transformation compared to Nipp. Thus, we conclude that it is easier to induce calli from the mature seeds of the varieties, including SN9816, LX1, Toyo, M31, and SN256.

\section{Transformation efficiencies of the six varieties are vary}

To investigate the transformation rates of the japonica rice varieties, 3-week-old secondary calli were infected with the Agrobacterium strain EHA105 harboring the pCambia 1301 vector. After 3 days of co-cultivation with Agrobacterium, transient expression efficiency of GUS was determined (Figure 4A). Intron-GUS is a convenient reporter gene since this gene is strongly expressed in rice cells but not in Agrobacterium cells that attach to the tissues (Chan et al. 1993). The rates of the GUS stained calli were analyzed (Figure 4B). The results showed that the GUS staining rate of SN9816 (59.17\%) was the highest, which was higher than that of Nipp (48.78\%). However, the rates of LX1 (20\%), Toyo (18.89\%), M31 (17.50\%), and SN265 (25.54\%) were lower than that of Nipp (48.78\%).

After 3-week selection in the N6D medium with $50 \mathrm{mg}$ $\mathrm{L}^{-1} \mathrm{Hyg}$ (N6D-S), the proliferation of Hyg-resistant calli was observed from all the varieties (Figure 4C). However, the rates of Hyg-tolerant calli or transgenic efficiencies of the varieties were different (Figure 4D), which is consistent with the previous results that transgenic efficiency is genotype dependent (Hiei et al. 1997, Hiei and Komari 2008, Saika and Toki 2010). The highest rate of Hyg-resistant calli was obtained from Toyo $(92.69 \%)$ and the lowest rate from SN265 (36.61\%). Toyo (92.69\%), SN9816 (91.60\%), and Nipp (82.96\%) had much more significant rates than LX1 (33.62\%), M31 (34.52\%), and SN265 (36.61\%) (Figure 4D). There was no significant difference among LX1, M31, and SN265. These results indicated that, in addition to Nipp, both SN9816 and Toyo were genotypes tolerant to Agrobacterium-mediated gene transfer, which gave rise to higher transformation efficiency after secondary selection on Hyg containing N6D-S medium (Figure 4D).
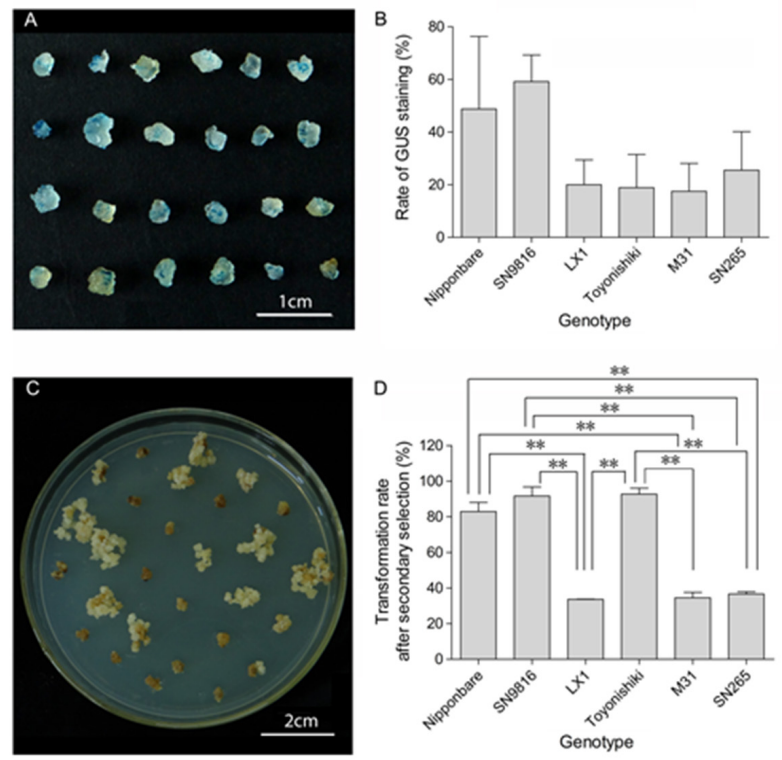

Figure 4. Agrobacterium-mediated transformation efficiency of the varieties in northern China. (A) Transient expression of 35S:intron-GUS after 3 days of co-cultivation with Agrobacterium. (B) The rate of GUS stained calli of the varieties. (C) The proliferation of the Hyg-tolerant calli in secondary selection medium, N6D-S medium. (D) Transgenic efficiencies of the varieties in northern China after secondary selection. Three biological replicates were performed. Similar results were obtained. The results from two or three replicates are shown. The values are the mean and standard deviations from three independent biological experiments. One asterisk $(*)$ represents significant difference $(P<0.05)$. Two asterisks $(* *)$ represent highly significant difference $(P<0.01)$. SN9816: Shen Nong 9816; LX1: Liao Xing 1; SN265: Shen Nong 265. White lines are the scale bar, representing $1 \mathrm{~cm}$ or $2 \mathrm{~cm}$ as indicated.

\section{The regeneration ability of the varieties differ from each other}

To explore the regeneration capability of the varieties, the secondary calli were transferred directly to the MSNK regeneration medium. The regeneration rates without transformation of the varieties on an inoculated callus basis were counted. The results revealed that the calli derived from all the varieties had the ability to regenerate (Figure $5 \mathrm{~A})$, but the rates of regeneration of the varieties differed. The regeneration rate of Toyo (73.99\%) was the highest, and that of M31 (22.93\%) was the lowest (Figure 5C). The regeneration rates of Toyo (73.99\%), Nipp (66.12\%), SN9816 (64.66\%), and SN265 (52.50\%) were significantly higher than LX1 (35.55\%) and M31 (22.93\%) (Figure 5C). The regeneration rate of SN265 was significantly higher than that of M31 (Figure 5C). No significant difference was detected among Toyo, Nipp, SN9816, and SN265, and between LX1 and SN265 (Figure 5C). These results suggest that there is a greater possibility of growing plants from the callus for Toyo, Nipp, SN9816, and SN265. However, the question remains as to whether the regeneration ability of 

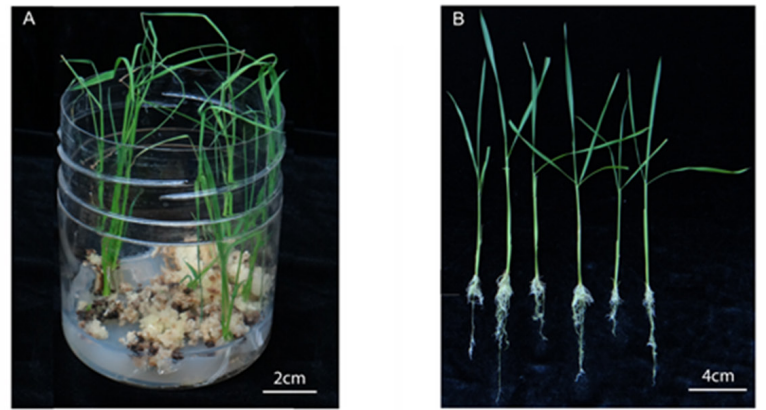

C
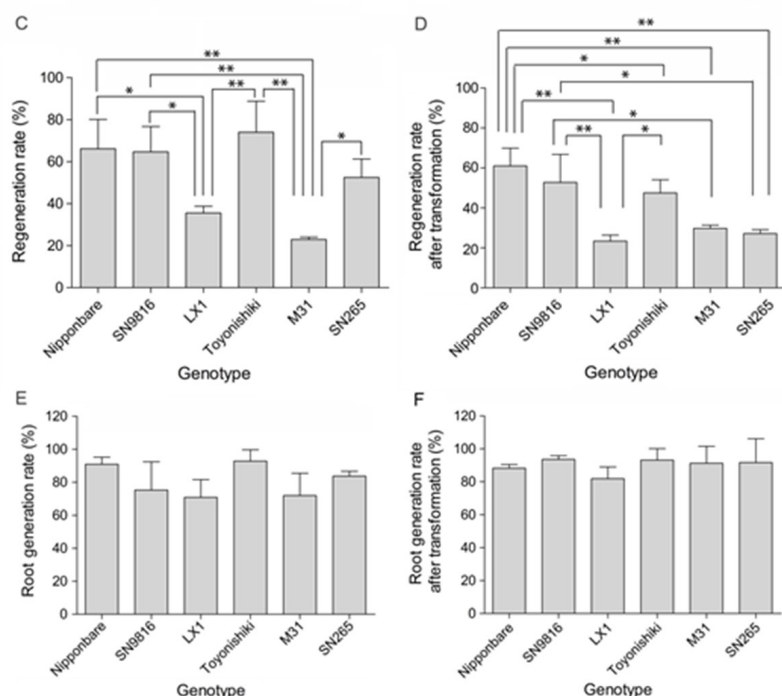

Figure 5. Regeneration ability of the varieties in northern China. (A) Regenerated shoots from the Hyg-tolerant tissues. (B) Plantlets with roots derived from Agrobacterium-mediated transformation. (C) Regeneration rates without Agrobacterium-mediated transformation of the varieties in northern China. (D) Regeneration rates with Agrobacterium-mediated transformation of the varieties in northern China. (E) The rate of root generation from the regenerated shoots without transformation. (F) The rate of root generation from the regenerated shoots with Agrobacteriummediated transformation. Three biological replicates were performed. Similar results were obtained. The results from two or three replicates are shown. The values are the mean and standard deviationsof two or three independent biological experiments. One asterisk $(*)$ represents significant difference $(P<0.05)$. Two asterisks $(* *)$ represent highly significant difference $(P<0.01)$. SN9816: Shen Nong 9816; LX1: Liao Xing 1; SN265: Shen Nong 265. White lines are the scale bar, representing $2 \mathrm{~cm}$ or $4 \mathrm{~cm}$ as indicated.

the varieties may be influenced by Agrobacterium-mediated transformation.

\section{Regeneration of the varieties is transgenic sensitive}

To investigate the response of regeneration to Agrobacterium-mediated transformation, the Hyg-tolerant calli of the varieties in N6D-S medium were transferred to the MS-NK regeneration medium. Regeneration ability was evaluated. The results showed that the regeneration rates after transformation of all the varieties were lower than the rate of calli subcultured in the regeneration medium without transformation (Figures 5C, D). The highest regeneration efficiency after Agrobacterium-mediated transformation was observed from the Nipp variety (60.98\%), and the lowest was from LX1 (23.33\%) (Figure 5D). The regeneration rates after Agrobacterium-mediated transformation of Nipp (60.98\%) and SN9816 (52.75\%) were significantly higher than those of LX1 (23.33\%), SN265 (27.14\%), and M31 (29.74\%) (Figure 5D). The rates of Nipp (60.98\%) and Toyo $(47.43 \%)$ were significantly higher than the rates of Toyo (47.43\%) and LX1 (23.33\%), respectively (Figure 5D). There was no significant difference between Nipp and SN9816 (Figure 5D). These results revealed that the regeneration of all six varieties was sensitive to Agrobacterium-mediated transformation, especially the Toyo variety (Figures 5C, D). Agrobacterium-mediated transformation reduced regeneration ability. However, the regeneration rates of Nipp and SN9816 were similar, at the level of $50 \%$ to $60 \%$, based on the number of Hyg-tolerant calli (Figure 5D).

\section{The rates of root growth of the regenerated shoots were similar among the varieties}

After regeneration, the regenerated shoots were transplanted to the root growth medium (MS-HF) to allow vigorous root growth (Figure 5B). The root-generating ability among the varieties was similar. No significant difference of root-emergence rates was observed among the varieties without or after Agrobacterium-mediated transformation (Figures 5E, F). We conclude that the regenerated shoots from the calli transformed or non-transformed varieties had
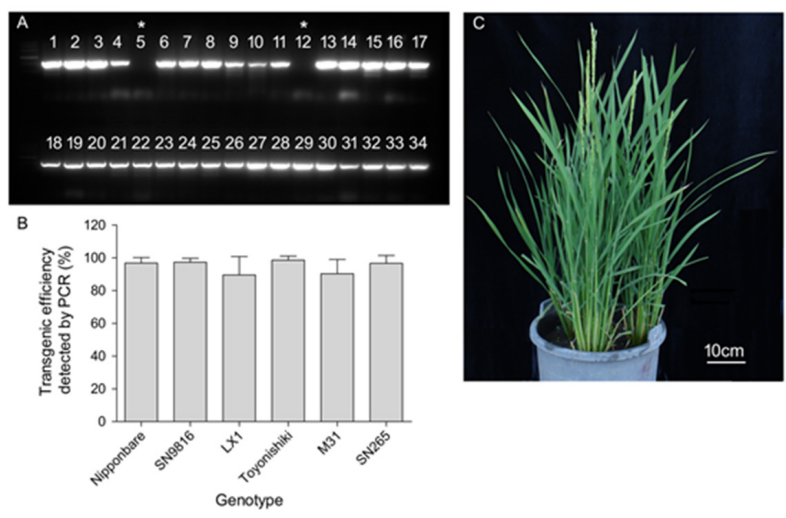

Figure 6. Detection of transgenic plantlets with PCR. (A) A gel image of PCR products with the HygF/HygR primer pair on Hyg-tolerant transgenic plantlets. The number represents the individual transgenic plantlets. The asterisk (*) labeled 5 and 12 represents negative plantlets. (B) Transgenic efficiency detected by PCR based on the number of the rooted regeneration plantlets. (C) Transgenic plants in heading stages. The white line is the scale bar, representing $10 \mathrm{~cm}$. 
similar ability to generate roots (Figures 5E, F).

To determine the rate of positive transgenic plants, Polymerase Chain Reaction (PCR) was performed on the genomic DNA of the candidate transgenic plants obtained with the primer pair ( $\mathrm{HygF} / \mathrm{HygR})$ of the Hyg gene in the pCambia 1301 binary vector (Figure 6A). The transgenic rates of the plants from the varieties were measured with the statistical method (Figure 6B). The transgenic rates of the candidates were from $93.75 \%$ to $98.58 \%$. No significant difference existed among the varieties. The transgenic plants were then transplanted to the soil to harvest seeds for the subsequent studies (Figure 6C).

To summarize, the transgenic efficiency of five varieties commonly grown in northern China were evaluated in this study and compared to Nipp. Though the rates of the secondary callus growth vary, the proliferation rates of primary and secondary calli of the varieties are similar, indicating that it is easier to induce calli from the mature seeds of the varieties (Figure 3). The transgenic efficiency of the varieties is different after secondary selection in the Hyg-containing N6D-S medium. The transgenic efficiencies of Toyo, Nipp, and SN9816 are similar, but much more highly significant than those of LX1, M31, and SN265 (Figure 4). Agrobacterium-mediated transformation suppresses regenera- tion of the varieties. The regeneration rates of Toyo, Nipp, and SN265 without transformation are higher than those of LX1 and M31 (Figure 5C). The regeneration rates after transformation of Nipp and SN9816 are higher than those of LX1, M31, and SN265 (Figure 5D). The regeneration of Toyo is hypersensitive to Agrobacterium-mediated transformation (Figures 5C, D). We may easily conclude that the performance of SN9816 during Agrobacterium-mediated gene delivery, including callus formation, transgenic efficiency, regeneration rate, and root generation rate, is similar to Nipp. Our data suggest that SN9816, which is the major cultivated super japonica variety with erect panicle, ideal plant architecture, and higher yield, is an elite germplasm for Agrobacterium-mediated transformation in northern China and the area at the same latitude. These results lay the sound foundation for us to generate the large number of T-DNA insertion plants needed for functional analysis of the rice genome and for breeding super japonica rice varieties with additional agronomic value.

\section{ACKNOWLEDGMENTS}

This study was supported by the National Natural Science Foundation of China (NSFC) [grant number 31371222] and the Doctoral Fund of the Ministry of Education of China (RFDP) [grant number 20132103110004].

\title{
Eficiência diferencial de transformação de variedades de arroz japonica desenvolvidas no norte da China
}

\begin{abstract}
Resumo - A produção de arroz japonica no norte da China desempenha um papel importante na segurança alimentar para o mundo. Transferência de genes mediada por Agrobacterium está se tornando uma abordagem poderosa para gerar germoplasma e desenvolver variedades. No entanto, a eficiência transgênica das variedades de arroz japonica no norte da China tem sido completamente desconhecida, o que obstrui o desenvolvimento da criação de transgênicos e a exploração das funções dos genes. Neste estudo, as eficiências da transgenia de seis variedades de arroz japonica desenvolvidas no Norte da China são avaliadas. As taxas de indução de calo primário e secundário das variedades são semelhantes. Contudo, a eficiência transgênica e a capacidade de regeneração das variedades são bem distintas. Foi estabelecida uma plataforma para a transformação das variedades de arroz e proposta uma variedade adequada, SN9816, para transferência de genes. SN9816 pode ser aplicada como um germoplasma-elite para melhoramento transgênico e pesquisa básica de biologia molecular no norte da China ou em uma área na mesma latitude.
\end{abstract}

Palavras-chave: Transformação mediada por Agrobacterium, eficiência de transformação, arroz japonica, no norte da China, Shen Nong 9816.

\section{REFERENCES}

Chan MT, Chang HH, Ho SL, Tong WF and Yu SM (1993) Agrobacteriummediated production of transgenic rice plants expressing a chimeric alpha-amylase promoter/beta-glucuronidase gene. Plant Molecular Biology 22: 491-506.

Hiei Y and Komari T (2008) Agrobacterium-mediated transformation of rice using immature embryos or calli induced from mature seed. Nature Protocols 3: 824-834.

Hiei Y, Komari T and Kubo T (1997) Transformation of rice mediated by Agrobacterium tumefaciens. Plant Molecular Biology 35: 205-218.
Hiei Y, Ohta S, Komari T and Kumashiro T (1994) Efficient transformation of rice (Oryza sativa L.) mediated by Agrobacterium and sequence analysis of the boundaries of the T-DNA. The Plant Journal 6: 271-282.

Jefferson RA, Kavanagh TA and Bevan MW (1987) GUS fusions: betaglucuronidase as a sensitive and versatile gene fusion marker in higher plants. EMBO Journal 6: 3901-3907.

Jung KH, An G and Ronald PC (2008) Towards a better bowl of rice: assigning function to tens of thousands of rice genes. Nature Review Genetics 9: 91-101. 
D Li1 et al.

Khush GS (1997) Origin, dispersal, cultivation and variation of rice. Plant Molecular Biology 35: 25-34.

Londo JP, Chiang YC, Hung KH, Chiang TY and Schaal BA (2006) Phylogeography of Asian wild rice, Oryza rufipogon, reveals multiple independent domestications of cultivated rice, Oryza sativa. Proceeding of the National Academy of Science of the United States of America 103: 9578-9583.

McLaren JS (2005) Crop biotechnology provides an opportunity to develop a sustainable future. Trends in Biotechnology 23: 339-342.

Nishimura A, Aichi I and Matsuoka M (2006) A protocol for Agrobacterium-mediated transformation in rice. Nature Protocols
1: $2796-2802$.

Raimondi JV, Marschalek K and Nodari RO (2014) Genetic base of paddy rice cultivars of Southern Brazil. Crop Breeding and Applied Biotechnology 14: 194-199.

Saika H and Toki S (2010) Mature seed-derived callus of the model indica rice variety Kasalath is highly competent in Agrobacterium-mediated transformation. Plant Cell Reports 29: 1351-1364.

Toki S, Hara N, Ono K, Onodera H, Tagiri A, Oka S and Tanaka H (2006) Early infection of scutellum tissue with Agrobacterium allows highspeed transformation of rice. The Plant Journal 47: 969-976. 